\title{
Neutrophil gelatinase associated lipocalin (NGAL) in early detection of nephropathy in type 2 diabetic Iraqi patients
}

\author{
Ahmed A. kahdem * \\ AliA.DyabAllawi** \\ Shaymaa Z. Nada * \\ Kismat M. Turki***
}

\author{
BSc, MSc (Clinical Chemistry) \\ MBChB,FRCP(London) \\ BSc, PhD (Clinical Biochemistry) \\ BSc, PhD(Clinical Chemistry)
}

\begin{abstract}
:
Background: Diabetic nephropathy is a common complication of diabetes mellitus type2. The neutrophil gelatinase Associated lippocallin (NGAL) is an ubiquitous protein consist of 178 amino acid. NGAL can be identified in plasma and urine starting 2- 4 hours after a kidney injury resulting changes in glomerular filtration and tubular reabsorption and with increased secretion in tubular epithelial cells.

Fac Med Baghdad 2017; Vol.59, No.1

Received: Jan. 2017

Accepted: Feb.2017

Objective: This study aimed to evaluate the role of serum Neutrophil Gelatinse Associated Lipocallin (NGAL) in early detection nephropathy.

Method : This study was conducted in Medical City, Baghdad Teaching Hospital during the period from December 2015to June 2016. The study included (90) subjects with age range between ( $30-56)$ years, divided in to three groups , 30 healthy control,30 type 2 diabetic mellitus patients with normal albuminuria and 30 type2 diabetic mellitus patients with micro albuminuria .Body mass index (BMI) was determined, serum NGAL, Fasting serum glucose (FBS),serum creatinine ,serum urea ,andglycated hemoglobin (HbA1c\%) were measured for each individual in addition to the concentration of albumin in urine and Glomerular Filtration Rate (eGFR) was calculated.

Results: The results of the present study showed that the levels of NGAL, FBS, HbA1c, urea creatinin, were significantly higher in micro albuminuria than normal albuminuria and healthy control. In diabetic patients with micro albuminuria, Serum NGAL shows significant positive correlation with FBS, $\mathrm{HbA} 1 \mathrm{c} \%$ and urine albumin. Serum NGAL shows significant positive correlation with duration of diabetes but significant negative correlation with GFR.

Conclusion:Serum NGAL increased markedly with the reduction of GFR.Serum NGAL is may be considered predictive marker for early detection of diabetic nephropathy in type 2 diabetes mellitus.

Keywords:diabetic mellitus, diabetic nephropathy,micro albuminuria, serum NGAL, HbA1c.
\end{abstract}

Introduction:

Diabetes mellitus is a heterogeneous group of multifactorial, polygenic syndromes characterized by an elevation of fasting blood glucose caused by a relative or absolute deficiency in insulin1. The chronic complication of diabetes mellitus affects many organ systems responsible for the majority of morbidity and mortality in both type 1 and type 2 diabetes. The chronic complications are divided in to macro vascular complications(coronary artery disease, peripheral arterial disease, and stroke)and micro vascular complications (diabetic nephropathy, neuropathy, and retinopathy) 2.Diabetic nephropathy is a common complication of diabetes mellitus type2. The mechanism includes changes in blood vessels that supply peripheral nerves and the metabolic disorder3.

*Department of Biochemistry, College of Medicine, University of Karbala, holy Karbala,

Email: (ahmed_albarkh@yahoo.com).

**Assistant professor and consultant in nephrology and kidney transplantation, College of medicine, University of Baghdad.

***Department of Biochemistry, College of Medicine, University of Baghdad.
Pathogenesis for diabetic nephropathy are complex and multiple; the early hemodynamic changes of glomerular hyper fusion and hyper filtration by leakage of albumin from the glomerular capillaries and structural changes as glomerular basement membrane thickening, glomerular hypertrophy,mesangial cell expansion,podocyte injury and loss, glomerularsclrosis,the clinical manifestations of nephropathy include a decrease in the glomerular filtration rate (GFR) and an increase in level of urinary albumin excretion 4. Early diagnosis of diabetic nephropathy important to presentation, therapy and decrease progression of renal disease 5.Micro albuminuria measurement for people with diabetes mellitus and people with GFR less than $60\left(\mathrm{ml} / \mathrm{min} / 1.73 \mathrm{~m}^{2}\right)$ and people with strong chronic kidney disease 7.The rate of urinary albumin excretion(UAER) in type 2 diabetes of $\geq 20 \mu \mathrm{g} / \mathrm{ml}$ strongly predicts end stage of renal disease and is also risk factor for development of cardiovascular disease ,the patient with type 2 diabetes and sever hypertension, the presence of micro albuminuria is a common finding 8 . Micro albuminuria is used as screening and diagnostic 
diabetic nephropathy, it is a marker of early kidney disease and also used as a predictor fordevelopment ofcoronary heart disease and mortality 9. The neutrophil gelatinase Associated lippocallin (NGAL) is an ubiquitous protein consist of 178 amino acid and molecularmass of approximately $25 \mathrm{kDa}$ from family lipocalins, this family associated with many biological processes; as inflammation and synthesis of prostaglandins.NGAL was initially identified by Allen and Venge in 1989 from human neutrophils and NGAL expressed at low levels in several human tissue as kidney,trachea, lunges, stomach and colon10.Human NGAL consist of a single disulphide - bridged polypeptide and the majority of NGAL is in a monomeric form and also occurs as dimers, trimmers and complex with neutrophil gelatinase .The monomeric NGAL form is secreted by injured kidney tubule epithelial cells and the dimeric form is the predominant form secreted by neutrophils.NGAL in urine derived from epithelial cells of the distal nephron11. NGAL can be identified in plasma and urine starting 2- 4 hours after a kidney injury resulting changes in glomerular filtration and tubular reabsorption and also increased secretion in tubular epithelial cells12.This study aimed to study the prediction of serum level of Neutrophil Gelatins Association lipocalin (NGAL) in early diabetic nephropathy in Iraqi diabetic patients.

\section{Subjects andMethods:}

This study was conducted in Medical City, Baghdad Teaching Hospital during the period from December 2015to June 2016.The study included (60) patient (30 male and 30 female) with type2 diabetic patients and 30 healthy controls. Their age range between (30 - 70)years. All patients have no history of liver disease, thyroid or other endocrine diseases through clinical interviewing. The subjects were divided in to three groups ;30 healthy control, 30 type 2 diabetic mellitus patients with normal albuminuria (albumin excretion in urine $<30 \mu \mathrm{g} / \mathrm{ml}$ ) and 30 type 2 diabetic mellitus patients with micro albuminuria(albumin excretion in urine $>30$ $\mu \mathrm{g} / \mathrm{ml}$ ).Urine sampling was performed by giving each subject a suitable disposable container with immediate freezing in the deep freeze at $-20 \circ$ C.The general physical, chemical and microscopic examination of urine were performed on another part of urine specimen to check for the final selection or exclusion of a subject in the study and blood sampling was performed at $8.00-11.00$ am in the fasting state for all the patients and controls and about seven milliliters of venous blood was obtained and divided into EDTA tube HbA1c test, and Plain tube, for serum collection in plain plastic tubes and stored at - 20oC.For all patients and controls, Hight and weight were measured for the calculation of (BMI) as a ratio of the weight to the hight $(\mathrm{kg} / \mathrm{m} 2)$. Blood pressure was measured three time and the average value was considered for data analysis .Fasting serum glucose (FSG) concentration was determined by using glucose oxidase method, $\mathrm{HbAlc} \%$ assay using the Siemens Dimension clinical chemistry system is an in vitro diagnostic assay for the quantitative determination of
$\mathrm{HbA1c}$ in human ,albumin in urine was measured by the fully-auto chemiluminescence immunoassay (CLIA) Analyzer MAGLUMI 1000),Serum urea and creatinine levels were determined using an enzymatic colorimetric methods,GFR was estimated using the modification of diet in renal disease (MDRD) abbreviated equation : $\{\mathrm{GFR}=186 \times($ serum creatinin $)-1.154 \times$ (age) $-.203 \times(0.742$ if female ) $\}$ and serum NGAL was measured using Cos bio kit ,enzyme-linked immunosorbent assay(ELISA).

Statistical analysis: result was expressed as mean \pm SD difference between the groups were analyzed by one-way analysis of variance (ANOVA).The correlation between various variables were calculated using the person correlation coefficient. p-value $<0.05$ were accepted as statistically significant .All statistical calculation were don using Statistical Package for the Social Science version 20(SPSS) .

\section{Results:}

The ANOVA test showed no statistically significant differencesinBMI $(p>0.05)$ between the studied groups. Systolic and Diastolic blood pressure showed statistically significant difference ( $p<0.001)$ among study groups. T test used showed statistically significant difference in the duration of diabetes among normal albuminuria and micro albuminuria diabetic patientgroups. Table (1) FSG level was significantly higher in micro albuminuria $(p<0.001)$ than normal albuminuria and healthy control.Glycated hemoglobin (HbA1c \%) was significantly higher in micro albuminuria(p $<0.05)$ than normal albuminuria and healthy control group. The levels of blood urea were significantly higher in micro albuminuria ( $p$-value $<0.05$ ) compared with normal albuminuria and healthy control group. Serum creatinine levels was significantly higher in micro albuminuria than normal albuminuria and healthy Group ( $\mathrm{p}<0.001)$. The mean value of (GFR) was significantly higher in micro albuminuria than normal albuminuria and healthy group $(\mathrm{p}<0.05)$.The mean value of serum (NGAL) was significantly higher in micro albuminuria than normal albuminuria and healthy control group $(\mathrm{p}<0.05)$.The person correlation analyses revealed the relationship between serum NGAL and (FBG),HbA1c \%, GFR , duration of the diabetes, and urine albumin in diabetic patients with micro albuminuria. Person correlation analysis showed that serum NGAL concentration has significant positive correlation with FSG $(r=0.398, p=0.02)$, and significant correlation between Serum NGAL and $\mathrm{HbA1c} \%(\mathrm{r}=0.394, \mathrm{p}=0.03$ )as shows figure (1). There was significant negative correlation between serum NGAL and GFR ( $r=-0.363, p=0.04)$ as shows figure (2), and there was significant positive correlation between serum NGAL and duration of diabetes $(\mathrm{r}=0.414, \mathrm{p}=0.02)$ as shows figure (3) .and show significant correlation between serum NGAL and albumin in urine $(\mathrm{r}=0.383, \mathrm{p}=0.03)$ as shows figure (4). 
Neutrophil gelatinase associated lipocalin (NGAL) for early diabetic nephropathy in

Iraqi type 2 diabetic patients.

Table (1) clinical characteristics of the studied groups.

\begin{tabular}{|c|c|c|c|c|}
\hline Characteristics & Healthy group & normal albuminuria & micro albuminuria & P-ANOVA \\
\hline Age (year) & $53.5 \pm 11.7$ & $53.7 \pm 11.5$ & $55.2 \pm 9.7$ & $>0.05$ \\
\hline BMI $\left(\mathrm{Kg} / \mathbf{m}^{2}\right)$ & $27.5 \pm 4.1$ & $29.21 \pm 4.8$ & $29.4 \pm 5.5$ & $>0.05$ \\
\hline BP(systolic)mm/Hg & $124.2 \pm 12.7$ & $133.0 \pm 17.1$ & $143.0 \pm 16.2$ & $<0.05$ \\
\hline $\mathrm{BP}($ diastolic)mm/Hg & $79.5 \pm 8.1$ & $87.0 \pm 5.8$ & $92.5 \pm 5.0$ & $<0.05$ \\
\hline Duration DM(year) & - & $5.9 \pm 2.0$ & $7.9 \pm 5.6$ & t-test $(<0.05)$ \\
\hline FBS(mg/dl) & $98.6 \pm 12.4$ & $197.6 \pm 63.0$ & $236 \pm 83.1$ & $<0.05$ \\
\hline HbA1c (\%) & $4.8 \pm 0.4$ & $7.5 \pm 0.7$ & $8.2 \pm 1.6$ & $<0.05$ \\
\hline Urine albumin $(\mu \mathrm{g} / \mathrm{ml})$ & $5.3 \pm 1.7$ & $18.7 \pm 4.9$ & $90.9 \pm 69.0$ & $<0.05$ \\
\hline Serum urea(mg/dl) & $34.6 \pm 8.3$ & $32.8 \pm 7.1$ & $43.2 \pm 17.0$ & $<0.05$ \\
\hline Serum creatinine $(\mathrm{mg} / \mathrm{dl})$ & $0.7 \pm 18.2$ & $0.8 \pm 21.7$ & $1.1 \pm 26.3$ & $<0.05$ \\
\hline $\operatorname{GFR}\left(\left(\mathrm{ml} / \mathrm{min} / \mathbf{1 . 7 3 m ^ { 2 } )}\right.\right.$ & $91.6 \pm 18.2$ & $85.6 \pm 21.7$ & $75.1 \pm 26.3$ & $<0.05$ \\
\hline Serum NGAL (ng/ml) & $4.9 \pm 2.1$ & $5.5 \pm 2.8$ & $6.2 \pm 1.6$ & $<0.05$ \\
\hline
\end{tabular}

ANOVA f- test $\mathrm{p}$-value $<0.05$ considered as significant $(\mathrm{S})$. $\mathrm{p}$-value $<0.001$ considered high significant (HS). And $\mathrm{p}$-value $>0.05$ considered nonsignificant, $\mathrm{SD}$ : standard deviation (mean $\pm \mathrm{SD}$ ), BMI: body mass index, BP: blood pressure.

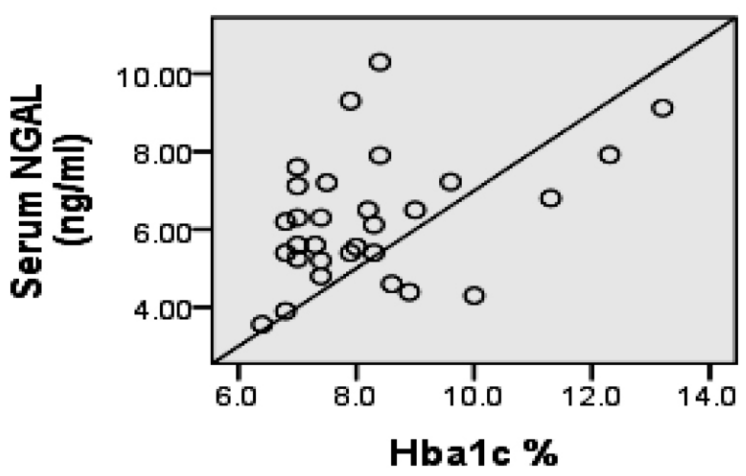

Figure (1) relationship between serum NGAL and HbA1c\%.

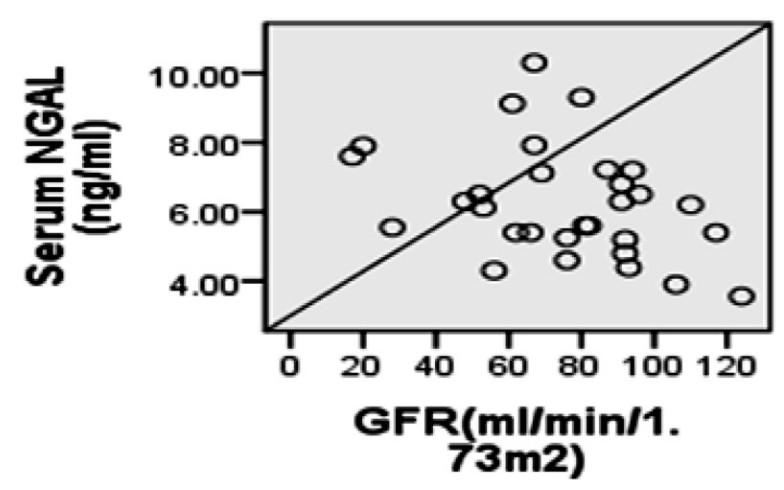

Figure (2) relationship between Serum NGAL and GFR

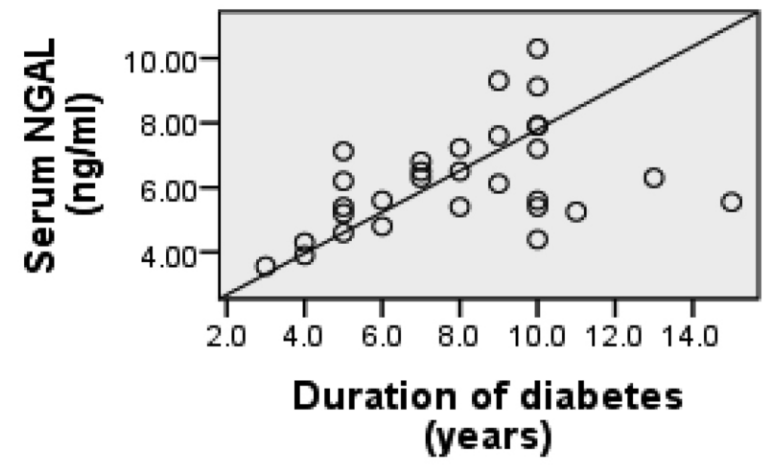

Figure (3) relationship between Serum NGAL and duration of diabetes

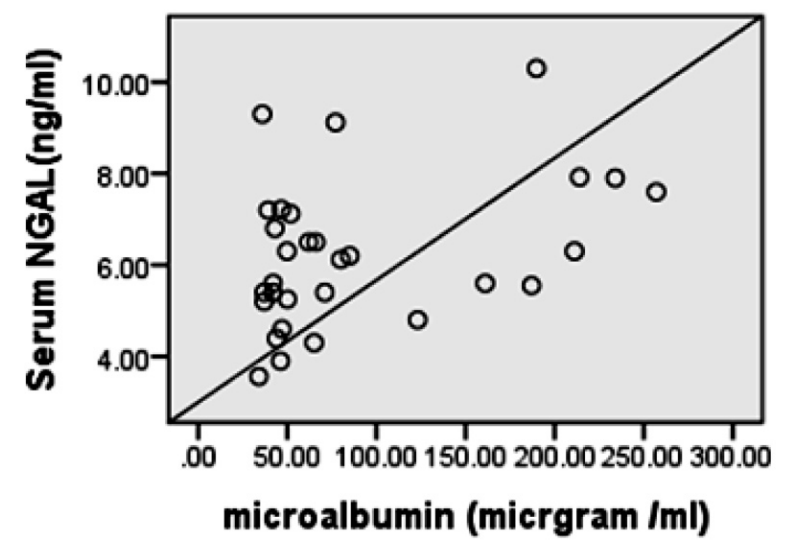

Figure (4) relationship between Serum NGAL and micro albumin. 


\section{Discussion:}

Diabetic nephropathy is the leading cause end stage of renal disease. Hyperglycemia causes increase hyper filtration and renal injury, in general hyperglycemia considered as the key initiator for kidney damage by activation of other metabolic pathways and formation or increase in oxidative stress 13. The presence of micro albuminuria considered as indicator or warning signal to renal and cardiovascular disease in patients with type 2 diabetes mellitus ,and considered also as the earliest marker of diabetic nephropathy and associated with significant glomerular damage. Studies showed that reduce risk factors lead to decrease level of micro albuminuria and decrease in renal and cardiovascular diseases 14. Studies showed that micro albuminuria dose not necessary reflect renal impairment in addition suggest that the early structure damage in both tubular structure and glomerular may be present in normal albuminuria 15.So there is a need to find biomarkers that help in identification the patients risk of the disease and Monitoring preventive and therapeutic effects.The results of this study showed that serum NGAL level is significantly increased in diabetic patients with microalbuminuria compared to diabetic patients with normal albuminuria and control group. This is in agreement with the finding of other study (Mahfouz et al 2016) 16.

.NGAL is a novel biomarker for early detection of acute renal failure because small molecule in size and resistance degradation ,NGAL excretion in serum and urine were found to be early predictive biomarker of acute kidney injury 17.NGAL level may be influenced by hypertension, renal diseases, inflammation condition; and other study stated that NGAL was effective in the early diagnosis of acute kidney injury 15.Other finding reported that hyperglycemia induce an incipient and alteration in the tubular handling of NGAL result in increased urinary excretion, suggesting the poor glycemic control of the diabetic patients lead to increase serum level of NGAL 18. Results of the present study show a statistically significant positive correlations between serum NGAL with fasting serum glucose and $\mathrm{HbA1c} \%$ of the diabetic patients with micro albuminuria .This is in agreement with the finding of other studies (Mohfaze et al 2006)16. The albumin excretion increased and has association with long term duration of diabetes mellitus and it is suggested that poor glycemic control play role in progression of diabetic nephropathy 19. Results of the present study show a statistically significant positive correlations between serum NGAL and urine albumin excretion this is in agreement with the finding of many studies(Bolingo et al 2009)20 .So that it is suggested that serum NGAL can be used as a marker of tubular injury precedes the appearance of micro albuminuria and as a marker of glomeruli injury 20. Results of this study show a statistically significant negative correlation between serum NGAL and of GFR in diabetic patients with micro albuminuria this is agreed with the finding of many studies( Donadio 2014 ) 21 There is statistically significant correlations between serum
NGAL and duration of diabetes mellitus with micro albuminuria This is in agreements with finding of other study( Mohfaze et al 216 )16and they found significant increase between serum NGAL and duration of diabetes.NGAL play role in Acute Kidney Injury in normal circumstances small amount may be found in plasma and urine 23.NGAL is filtered from plasma mainly through glomerular filtration and after filtration the NGAL is reabsorbed by proximal tubule cells by endocytosis via the megalin system 22. When proximal tubule is damaged by tubular necrosis filtered NGAL can escape tubular reabsorption and secreted in urine , high serum NGAL and high filtered saturates tubular capacity that lead to increase urinary and serum NGAL. So serum NGAL and urinary NGAL have been indicated as marker of acute kidney injury and accumulation hyperglycemia and hypertension induce an incipient and specific alteration in the tubular handling of NGAL resulting increased in plasma and urine23. So it is suggested the long duration of diabetes and poor glycemic control lead to increase NGAL in serum and urine.

\section{Authors contributions:}

Ahmed.A. kadhem : Student

Ali A.Dyab Alawi : Study design

Kismet M.Turki : Supervisor

ShaymaaZ.Nada : Supervisor

\section{Conclusion:}

Poor glycemic control is characteristic of diabetes patients with micro albuminuria. In diabetic patients with micro albuminuria the serum NGAL increase markedly with the reduction of amount GFR.Serum NGAL is may be considered as predictive marker of renal injury correlated with renal function decline in type 2 diabetes mellitus also Serum NGAL considered as biomarkers for early detection of diabetic nephropathy in type 2 diabetes mellitus.

\section{References:}

1. Ferrier DR. Biochemistry. Lippincott Williams \& Wilkins; 2013 May 24.

2. Fowler MJ. Microvascular and macrovascular complications of diabetes. Clinical diabetes. 2008 Apr 1;26(2):77-82

3. Zakerkish M, Shahbazian HB, Shahbazian H, Latifi SM, Aleali AM. Albuminuria and its correlates in type 2 diabetic patients. Iranian journal of kidney diseases. $2013 \mathrm{Jul}$ 1;7(4):268.

4. Bakris GL. Recognition, pathogenesis, and treatment of different stages of nephropathy in patients with type 2 diabetes mellitus. InMayo Clinic Proceedings 2011 May 31 (Vol. 86, No. 5, pp. 444-456). Elsevier.

5. Satirapoj B, Siritaweesuk N, Supasyndh O. Urinary angiotensinogen as a potential biomarker of diabetic nephropathy. Clinical kidney journal. 2014 Aug 1;7(4):354-60.

6. Josipović J, Katičić D, Pavlović D. Dijabetičkanefropatija: 
dijagnostika, prevencija $i$ liječenje. MEDIX. 2013 Nov 15;19(107/108).

7. Viberti $G$, Wheeldon NM, MicroAlbuminuria Reduction With VALsartan (MARVAL) Study Investigators. Microalbuminuria reduction with valsartan in patients with type 2 diabetes mellitus a blood pressure-independent effect. Circulation. 2002 Aug 6;106(6):672-8.

8. Barbieri M, Rizzo MR, Fava I, Sardu C, Angelico N, Paolisso P, Abbatecola A, Paolisso G, Marfella R. Awaking blood pressure surge and progression to microalbuminuria in type 2 normotensive diabetic patients. Journal of diabetes research. 2016 Jan 1;2016.

9. Xia F, Liu G, Shi Y, Zhang Y. Impact of microalbuminuria on incident coronary heart disease, cardiovascular and all-cause mortality: a meta-analysis of prospective studies. International journal of clinical and experimental medicine. 2015;8(1):1.

10. Al-Refai AA, Tayel SI, Ragheb A, Dala AG, Zahran A. Urinary neutrophil Gelatinase associated lipocalin as a marker of tubular damage in type 2 diabetic patients with and without albuminuria. Open Journal of Nephrology. 2014 Mar 7;2014.

11. Haase-Fielitz A, Haase M, Devarajan P. Neutrophil gelatinaseassociated lipocalin as a biomarker of acute kidney injury: a critical evaluation of current status. Annals of Clinical Biochemistry: An international journal of biochemistry and laboratory medicine. 2014 May 1;51(3):335-51.

12. Firu SG, Streba CT, Firu D, Tache DE, Rogoveanu I. Neutrophil Gelatinase Associated Lipocalin (NGAL)-a biomarker of renal dysfunction in patients with liver cirrhosis: Do we have enough proof?. Journal of medicine and life. 2015;8(Spec Issue):15.

13. Sun YM, Su Y, Li J, Wang LF. Recent advances in understanding the biochemical and molecular mechanism of diabetic nephropathy. Biochemical and biophysical research communications. $2013 \mathrm{Apr}$ 19;433(4):359-61..

14. Khalef. H Youssef,.Association between Microalbuminuria and glycosylated hemoglobin, some oxidative stress biomarkers and Atherogenicity in type 2 diabetes women.College of medicine and committed of postgraduate studies at .AL.mustansiriya university 2015 .

15. Papadopoulou-Marketou N, Skevaki C, Kosteria I, Peppa M, Chrousos GP, Papassotiriou I, Kanaka-Gantenbein C. NGAL and cystatin C: two possible early markers of diabetic nephropathy in young patients with type 1 diabetes mellitus: one year follow up. Hormones (Athens). 2015 Apr 1;14(2):232-40.

16. Mahfouz MH, Assiri AM, Mukhtar MH. Assessment of Neutrophil Gelatinase-Associated Lipocalin (NGAL) and RetinolBinding Protein 4 (RBP4) in Type 2 Diabetic Patients with Nephropathy. Biomarker insights. 2016;11:31..

17. Shaker O, El-Shehaby A, El-Khatib M. Early diagnostic markers for contrast nephropathy in patients undergoing coronary angiography. Angiology. 2010 Nov 1;61(8):731-6.

18. Gordillo, R., Ahluwalia, T. and Woroniecki, R.,.Hyperglycemia and acute kidney injury in critically ill children. International
Journal of Nephrology and Renovascular Disease 2016, 9, p.201. 19. Zakerkish M, Shahbazian HB, Shahbazian H, Latifi SM, Aleali AM. Albuminuria and its correlates in type 2 diabetic patients. Iranian journal of kidney diseases. $2013 \mathrm{Jul}$ 1;7(4):268.

20. Bolignano D, Lacquaniti A, Coppolino G, Donato V, Campo S, Fazio MR, Nicocia G, Buemi M. Neutrophil gelatinase-associated lipocalin (NGAL) and progression of chronic kidney disease. Clinical Journal of the American Society of Nephrology. 2009 Feb 1;4(2):337-44.

21. Donadio C. Effect of glomerular filtration rate impairment on diagnostic performance of neutrophil gelatinase-associated lipocalin and B-type natriuretic peptide as markers of acute cardiac and renal failure in chronic kidney disease patients. Critical Care. 2014 Feb 28;18(1):1.

22. Van Deursen, V.M., Damman, K., Voors, A.A., van der Wal, M.H., Jaarsma, T., van Veldhuisen, D.J. and Hillege, H.L. Renal tubular damage. Comorbidity in heart failure2013, p.52.

23. Singer E, Markó L, Paragas N, Barasch J, Dragun D, Müller DN, Budde K, Schmidt Ott KM. Neutrophil gelatinase associated lipocalin: pathophysiology and clinical applications. Actaphysiologica. 2013 Apr 1; 207(4):663-72. 